\title{
Manuscript Shapes Generated by Novel Bi-Axis Control Algorithm Based on A Mathematical Handwriting Model
}

\author{
Imene Mahmoud, Ines Chihi, Afef Abedlkrim, Mohamed Benrejeb \\ University Tunis EL Manar, National Engineering School of Tunis (ENIT), Tunisia \\ Department of Electrical Engineering, Automation laboratory LARA.
}

\begin{tabular}{l} 
Article Info \\
\hline Article history: \\
Received \\
Revised \\
Accepted
\end{tabular}

Keyword:

$(\mathrm{x}, \mathrm{y})$ Plane

Algorithm

Bresenham bi-axis Control

Cursive Arabic Letters.

Electromyography

Human Handwriting Motion

Signals $E M G$ Recursive Least

Square Algorithm

Velocity

\section{Corresponding Author:}

Imene Mahmoud,

University Tunis El Manar,

National Engineering School of Tunis (ENIT), Tunisia.

Departement of Electrical Engineering

BP 37, 1002, The Bélvédère , Street Bashir Salem Belkhiria , University Campus , 1002, Tunis, Tunisia

Email: mahmoudimene89@gmail.com

\begin{abstract}
Handwriting movement is one of the most complex activities of human motions. It's a blend of kinesthetic, cognitive, perceptual and motor components. The study of this biological process shows that bell-shaped velocity profiles are generally observed in the handwriting motion. In this paper, an identification technique, based on Recursive Least Square algorithm (RLS), is proposed to identify the pen-tip movement in human handwriting process, by using input and output data which present EMG signals and velocities according to $\mathrm{x}$ and $\mathrm{y}$ coordinates. Using the estimated coordinates that have resulted from the velocity model; we propose a novel algorithm to generate handwritten graphic traces, which is inspired from the idea of tracing circles by Bresenham bi-axis control algorithm. The effectiveness of this approach should be observed on predicting cursive Arabic letters and Arabic word written on $(\mathrm{x}, \mathrm{y})$ plane, these shapes constituting a recorded experimental basis.
\end{abstract}

Copyright () 2015 Institute of Advanced Engineering and Science. All rights reserved.

\section{INTRODUCTION}

As well as oral language, handwriting is regarded as a communication tool and essential to educational, professional and social inclusion. Writing is a complex and fast motor skill requiring a certain level of language development, mastery of graphic space and a certain degree of motor development, and emotional praxis. Writing is basically an activity and individual characteristic as well as the voice and face of one person. It reflects the mental and physical state of writer, [29] and [30]. It also requires good coordination and careful arrangement of several factors such as the generation of nerve impulses, movements of the upper limbs, etc. Handwriting velocity has also attracted the attention of many researchers who considered it as a means of distinguishing between the writing of one person and that of another. They proved that the handwriting speed is a fundamental characteristic for modeling the handwriting system. Plamondon confirmed that the velocity profiles of this process are approximately bell shaped, [1], [2], [3], [4], [5], [6], [7], [8], [9], [10].

Scientists fed their entire lifetime in developing technologies that would act as a substitute to hide physical impairment. Past achievements infer that there were devices which when coupled with a physically challenged person, would assist in erasing the traces of the abnormality. But still the originality of humans retains its color. No device could do the job with so much perfection as a human does. And every human, physically able or disabled, is unique. Why can't approaches imitate human's uniqueness of movements, especially handwriting motion, [11], [12]. 
Various conventional and unconventional approaches have been developed in the literature to characterize handwriting process and its control system [13], [14], [15], [16], [17], [18], [19], [20], [21], [22]. As a first analysis, Van Der Gon developed a mathematical model characterizing the phenomenon, [23]. An electronic version is then proposed by Mc Donald who considered the system of handwriting as a mass moving in a viscous medium [24]. This movement is described by a linear differential equation of second order. A model governed by two nonlinear differential equations of the second order was developed by Yasuhara, who joined the effect of the frictional force between the pen tip and the writing surface, then he identified and decomposed rapidly writing system [25], [26]. From this model, Iguider developed two approaches; the first is used to extract the pulsations control [27], and the second to recognize the cursive Arabic script [28]. Edelman and Flash have developed, in 1987, a model based on the study of the trajectories of the hand [29].

Using unconventional approaches, several models are proposed for the characterization of the process of handwriting. These models are based on the concepts of artificial neural networks, the fuzzy logic, genetic algorithms, etc., [30]. Other approaches based on control algorithm were proposed to develop mobile limb models, [31], [32], [33], [34], [35].

The different models proposed in the literature to characterize the human handwriting process have contributed to the characterization of a limited number of handwritten shapes. Indeed, the generation of handwriting traces needs more precision. Particularly, Arabic letters represent much more curvature so the follow of this letters or their recognition is more complex.

Based on this principle and using Bresenham algorithm, we propose, in this paper, a bi-axis control approach, to generate some handwriting cursive Arabic letters and geometric forms

The following paper is organized as follows. Section II presents the experimental approach used in this paper. The next section emphasizes on the identification of the bell-shaped velocity profile relative to the handwriting movement. Then simulation's results are applied to different Arabic letters and simple geometrical forms relative to many writers. Section IV represents the novel proposed algorithm to generate handwriting traces. The discussion of the new algorithm and numerical results are represented in section $\mathrm{V}$. The conclusion and some perspectives are in section VI.

\section{MATHERIALS AND METHODS}

\subsection{Experimental approaches and data basis}

The production of graphic traces is treated as a natural process of a complex cognitive event. Indeed, the brain processes the location information of the pen tip, sent by the eyes. This information is analyzed and evaluated by an intelligent control system, to send an order to the muscles of the forearm to move the hand to the desired new position [36]. During the act of writing, the movements performed can be described like displacements in two-dimensional space of the writing plan. In the literature, multiple researches proved that the natural component of the graphic trace corresponds to space displacements of the pen during the formation of the trajectory [37], [38], [39]. In spite of the complexity of the effectors systems including the articulations of the shoulder, the elbow, the wrist and the hand, for a total of forty three muscles, the study conducted by Yasuhara identified four principal muscles for the hand control. From this analysis, an experimental study carried in [40], [41], [42], has recorded Electromyography signals (EMG) during the act of writing. Starting from the surface electrodes used per pair and having a common mass, the experimental study allowed recording the pen-tip coordinates moving on $(\mathrm{x}, \mathrm{y})$ plane and electromyography signals during the writing time. These signals can be measured easily on the skin surfaces at some muscle regions. However, it can only extract the global features of muscle movements because it is the temporally and spatially accumulated signal from many motor units. Therefore, it is not guaranteed that each EMG signal contains the information about the motor commands on writing. We consequently assume that the adequate information to represent the time series of the motor commands can be extracted with some suitable signals obtained by processing the EMG signals measured from several muscles related with handwriting movement [43], [44], [45].

In order to measure the pen-tip movementon ( $\mathrm{x}, \mathrm{y})$ plane and the EMG signals of forearm muscles on writing processes, an experimental approach was proposed to construct a measuring system shown in Figure 1. Indeed, EMG signals are obtained from the two most active muscles of the forearm, namely the "abductor pollicis longus" and the "extensor capri ulnaris" which are the most active and are opposed in movement; when one contracts the other extends.

This experimental approach requires a digital pen tablet (WACOM, KT-0405-RN), a PC to display and memorize the traces of the pen-tip movement and its pressure force $\mathrm{P}$ on the writing support. The EMG signals are measured at the disposable electrodes (MEDICOTEST, Blue Sensor N-00-S) adhered on the skin of the subject's writing forearm, and are recorded into the data recorder (TEAC, DR-C2) through the 
preamplifiers (TEAC, AR-C2EMG1). The sampling rate for measuring the EMG signals is $1000 \mathrm{~Hz}$ and that for measuring the pen-tip movement is about $500 \mathrm{~Hz}$. Those measuring data are synchronized by sending a step signal from the parallel interface port on the PC to the data recorder.

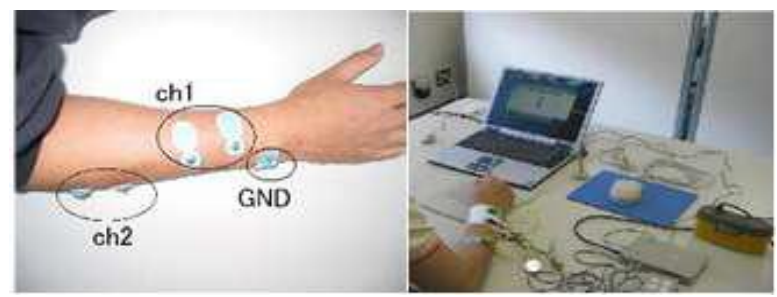

Figure 1. Magnetization as a function of applied field.

\section{THE PROPOSED ALGORITHM TO GENERATE HANDWRITING TRACES}

Meunlenbroek also showed that the generation of vertical lines is faster than horizontal and the movement from top to bottom is faster and precise lines that movement from bottom to top [46]. Based on this analysis, we chose, participants, in the experimental approach, that are used to produce Japanese writing systems, having a vertical direction of travel or an horizontal one from left to right which is different from Arabic writing systems. In this experiment, the participants have written geometric forms and Arabic letters, which influences, obviously, on the velocity and the preferred direction of the writers. In this case the production of geometric shapes is faster. These writers have drawn eight basic geometric shapes, and three cursives Arabic letters. Indeed, Arabic letters present more curvature than these geometric shapes and the generation of those letters is much more complex.

In this section, we propose a new algorithm to generate Arabic letters and complex geometric shapes using bi-axis control technique. In this context there are several algorithms proposed in the literature including Bresenham algorithm and Digital Differential Analyzer algorithm (DDA). The proposed algorithm of handwriting shapes' generation is inspired from Bresenham algorithm. This last one is also named "Midpoint", because of its high efficiency and ease of implementation and it is widely used in the computer graphic raster displaying device. DDA has the following problems: Accumulation of round-off errors can make the pixilated line drift away from what was intended and the rounding operations and floating point arithmetic involved are time consuming, [47].

\subsection{Analysis of handwriting velocity to identify handwriting process from EMG signals}

We assume that the handwriting velocity plays a significant role in the control and genesis of the human handwriting process and that the central nervous system may take this information into account in the reconstruction of the pen-tip trace. Significantly, the electromyography (EMG) signals measured on the skin surface of a writing forearm contain the sufficient information to illustrate the motor commands of the handwriting process. Velocity profiles corresponding to different measurements of the pen-tip shifts are presented to prove the invariance of these profiles which takes place as bell-shaped. The correlation between the velocity profile $\mathrm{V}(\mathrm{k})$ and the trajectory of the pen-tip according to $\mathrm{x}$ and $\mathrm{y}$ axes is then analyzed. The velocity of the pen-tip movement is calculated as follow:

$$
\begin{aligned}
V(k) & =\sqrt{V_{x}(k)^{2}+V_{y}}(k)^{2} \\
V_{x}(k) & =\frac{x(k+1)-x(k)}{t(k+1)-t(k)} \\
V_{y}(k) & =\frac{y(k+1)-y(k)}{t(k+1)-t(k)}
\end{aligned}
$$

$V \quad$ : pen-tip velocity

$V_{x}, V_{y}$ : pen-tip velocities according to $X$ and $y$ axis respectively

$x, y$ : pen-tip displacement according to $x$ and $y$ axis respectively

$t(k)$ : time of handwriting movement

\subsection{Case of simple geometric movements}

The experimental basis avows the study of two simple graphic forms namely "circle" and "triangle", Figure 2.a and 2.c. The Figure 2.b illustrates that the velocity shape of the geometric form "circle" conforms 
to a superposition of bell-shapes and the velocity profile of the geometric form "triangle" combines three bell shapes which corresponds to the three segments of the triangle, figure 2.d.
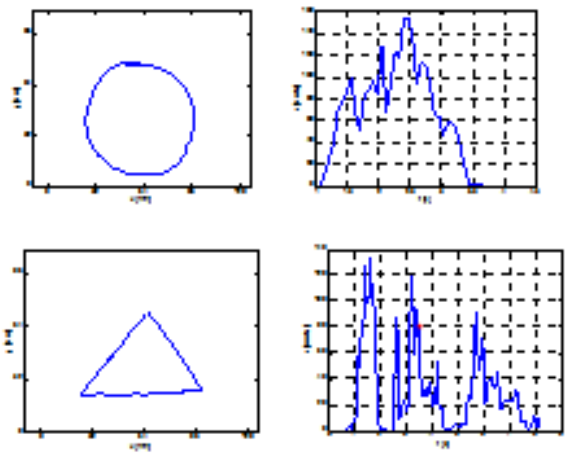

Figure 2. Form and velocity profiles of geometric forms, circle and triangle

\subsection{Case of Arabic letters}

The velocity profile of the Arabic letter "SIN" is shown in figure 3.
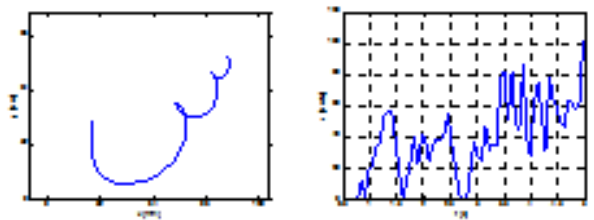

Figure 3. Form and velocity profile of the Arabic letter "SIN"

The handwriting velocity of "SIN" has three main ranges, diverse in durations and amplitudes, constituted by a velocity of bell-shaped forms that corresponds to three primitives of the considered letter: two small half circles and a larger third one. The velocity of handwriting trajectory shows some progressions of maxima. Zero value indicates the beginning, the end, as well as the change of movement direction. In other words, the characteristic of the letter "AYN" allows determining two areas that are formed by a superposition of bell-shaped forms that corresponds to the two already existing primitives of the letter, consisting of two halves of circles. Therefore, the writing velocity can be understood as a superposition of bells distributed by field with different durations and amplitudes. Each zone corresponds to a specific primitive of the written letter.

\subsection{Proposed handwriting velocity model}

The parametric identification is by definition, an experimental approach of determining mathematical model parameters of a given process and that generates an output response, similar to the real system. As a result, this technique is based on the prediction error, which links the output of the process and the output predicted by the model. In order to minimize the prediction error, the model parameters are to be modified and altered at each sampling time through the use of an algorithm which estimates the model parameters. In the literature numerous recursive and non-recursive algorithms are presented. The first type of algorithm can be executed on real time and requires less memory resources in terms of calculations. In this section, the model's structure is firstly proposed. Then, the Recursive Least Square algorithm is used in order to identify the handwriting process, through the use of velocity information [13].

The suggested mathematical model which characterizes the handwriting velocity is a fourth order model input delayed $X$ and $Y$ velocities, $V_{x}$ and $V_{y}$ at $k-1, k-2, k-3$ and $k-4$ instants, in addition to inputs related to EMG signals delayed at $k, k-1, k-2$ and $k-3$ model :

The outputs are $V_{x}$ and $V_{y}$ at $\mathrm{k}$ instant, figure 4. The following equations characterize the proposed 


$$
\begin{aligned}
& V_{x e}(k)=\sum_{i=1}^{4}-\left[a_{i x}(k) V_{y e}(k-i)+b_{i x}(k) V_{x e}(k-i)\right] \\
& +\left[c_{i x}(k) e_{1}(k-i+1)+d_{i x}(k) e_{2}(k-i+1)\right] \\
& V_{y e}(k)=\sum_{i=1}^{4}-\left[a_{i y}(k) V_{x e}(k-i)+b_{i y}(k) V_{y e}(k-i)\right] \\
& +\left[c_{i y}(k) e_{1}(k-i+1)+d_{i y}(k) e_{2}(k-i+1)\right]
\end{aligned}
$$

\subsubsection{Identification technique}

Recursive Least Squares algorithm (RLS) is used for the handwriting velocity process identification [14]. It offers a good convergence rate, especially for highly correlated input signals. The efficiency of this algorithm in terms of convergence rate, tracking and stability depends on the forgetting factor. In this study, we chose a fixed forgetting factor, equal, in this work, to 0.95

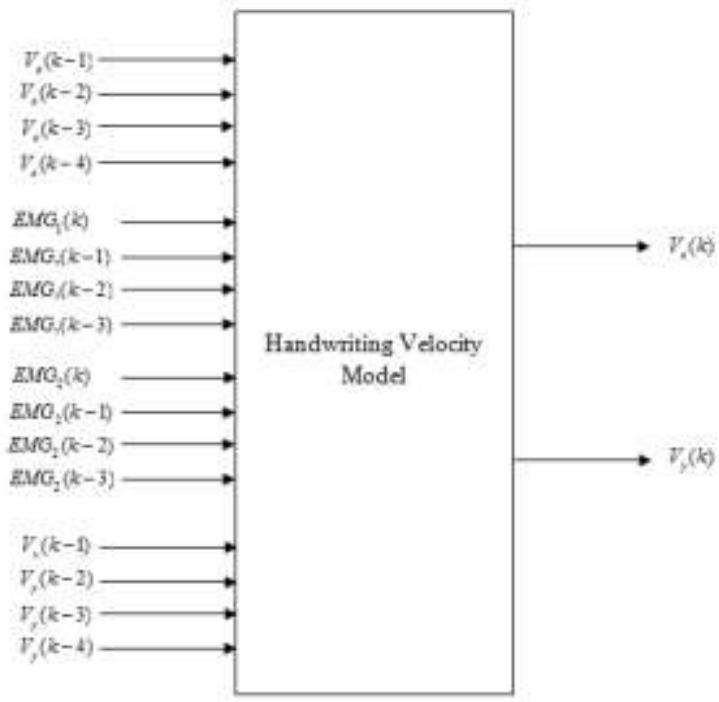

Figure 4. New proposed structure of handwriting velocity model

RLS algorithm performs the following operations to update the parameters of the researched model, [15].

$$
\begin{aligned}
\hat{\theta}(k)= & \hat{\theta}(k-1)+P(k) \sum_{i=n+1}^{k} y(i) \Psi(i) \\
P(k)= & P(k-1)-\frac{P(k-1) \Psi(k) \Psi^{T}(k) P(k-1)}{1+\Psi^{T}(k) P(k-1) \Psi(k)} \\
& \varepsilon(k)=y(k)-\hat{\theta}(k-1) \Psi(k)
\end{aligned}
$$

With

$$
\begin{aligned}
& \hat{\theta} \quad \text { : vector of estimated parameters, } \\
& \mathrm{P}(\mathrm{k}) \text { : adaption matrix, }
\end{aligned}
$$


$y(\mathrm{k})$ : actual output the sistem to identify,

$\psi(\mathrm{k})$ : observation matrix,

$\varepsilon(\mathrm{k})$ : estimated error.

The model structure *used to identify the handwriting system dynamics for multi-inputs-multioutputs is given as follows:

$$
\begin{gathered}
V_{\mu \alpha}=\psi_{\alpha}^{T} \theta_{\alpha}+\varepsilon_{\nu} \\
V_{y c}=\psi_{y}^{T} \theta_{y}+\varepsilon_{y}
\end{gathered}
$$

$\varepsilon_{\mathrm{x}}$ and $\varepsilon_{\mathrm{y}} \quad$ : error vectors, relative to the velocities according to $x$ and $y$ movements respectively $\psi_{x}^{T}$ and $\psi_{y}^{T}$ : Matrices whish elements are the delayed inputs and outputs components relative to the velocities according to $x$ and $y$ movements respectively.

Using the estimated parameter's values, the new proposed handwriting velocity model is as follows:

$$
\begin{aligned}
& V_{x e}(k)=\sum_{i=1}^{4}-\left[\hat{a}_{i x} V_{y e}(k-i)+\hat{b}_{i y} V_{x e}(k-i)\right] \\
& +\left[\hat{c}_{i x} e_{1}(k-i+1)+\hat{d}_{x} e_{2}(k-i+1)\right] \\
& V_{y e}(k)=\sum_{i=1}^{4}-\left[\hat{a}_{i y} V_{x e}(k-i)+\hat{b}_{i y} V_{y e}(k-i)\right] \\
& +\left[\hat{c}_{i y} e_{1}(k-i+1)+\hat{d}_{p y} e_{2}(k-i+1)\right]
\end{aligned}
$$

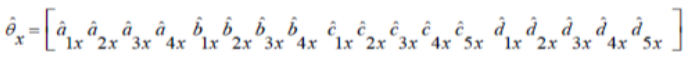

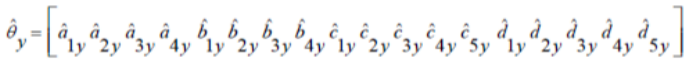

The predicted parameters of velocities vectors $\hat{\theta}_{x}$ and $\hat{\theta}_{y}$ according to ${ }^{x}$ and ${ }^{y}$ movements, respectively, will take the following forms:

$$
\begin{aligned}
& \hat{a}_{i x,}, \hat{b}_{i x,}, \hat{c}_{i x,} \hat{d}_{i} \text { : estimated parameters relative } \\
& \text { to the estimated velocities } \\
& V_{x e} \text { and respectively } \\
& \hat{a}_{i y}, \hat{b}_{i y}, \hat{c}_{i y,}, \text { : estimated parameters relative to the } \\
& i \quad: 1,2,3 \text { or } 4 \text {. }
\end{aligned}
$$

Figure 5 shows some examples of the comparison between the real trajectory of handwriting velocity and the reconstructed velocity trajectory with the same starting point. In addition, good concordances between the saved letters and the letters obtained from the proposed model are shown.

The solid line presents the recorded experimental data and the dotted line is relative to the answer given by the estimated parameters of the obtained model. 


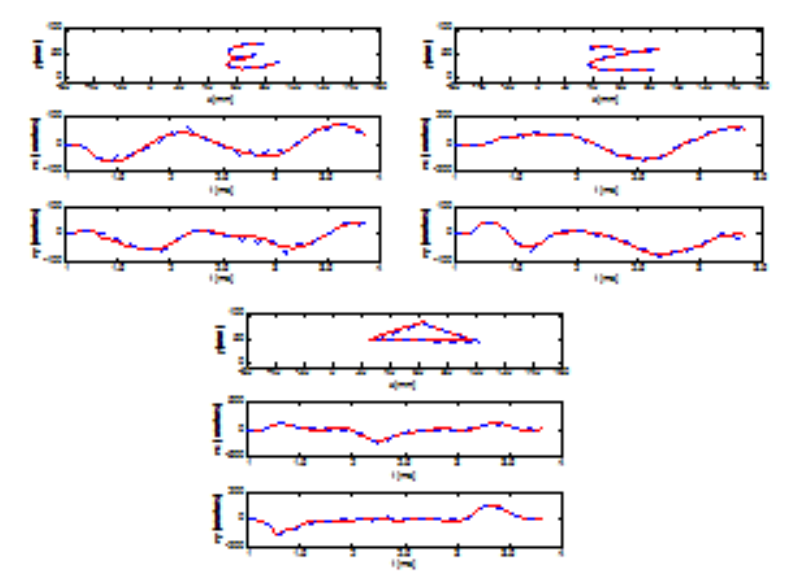

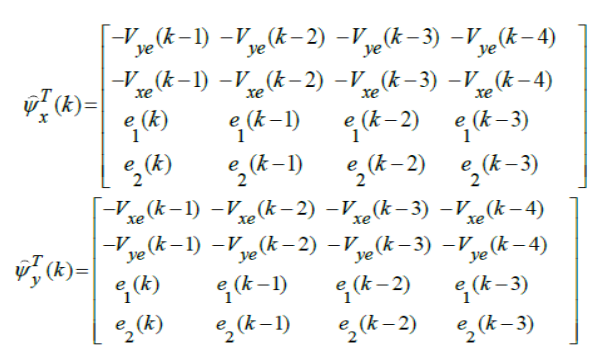

Figure 5. Obtained identification results (form, velocities according to $x$ and to $y$ axis)

Proposed in this section, are two types of validation of the proposed handwriting velocity model. The first one predicates the pen-tip trajectories in the mono writer case. This result is in the integration of the parameters of a model characterizing a letter or a geometric form with data saved from another example of the same kind of drawing trace and of the same writer. The second one is a prediction in the multi writer case. In order to do so, we did apply the experimental recorded input data of an Arabic letter or a basic geometric form for a first writer with parameters of another model characterizing the same pen-tip trace of another writer.

\subsubsection{Validation of the proposed handwriting velocity model : Monowriter case}

Recorded data relative to one model of graphic trace are here applied to different models for the same writer and the same type of the trace.

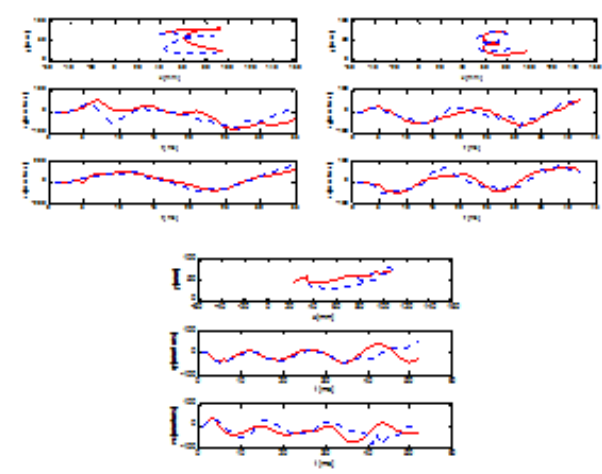

Figure 6. Some validities' results in monowriter case (form, velocities according to $x$ and to $y$ axis)

The compatibility between the prediction results of pen-tip velocity and the experimental data are shown in figure 6. Dotted lines show recorded results and solid lines illustrate predicted ones. Ultimately, for 
various types of Arabic letters and geometric drawings by the same writer, our proposed model confirms the possibility of direct prediction on handwriting velocities and traces from ${ }^{E M G}$ signals.

\subsubsection{Validation of the proposed handwriting velocity model : Multiwriter case}

The aim of this section is to propose one model for the same types of trace but for two different writers. This is obtained by applying EMG data recorded for a trace written by writer1 into a model of the same type of drawing relative to a writer2. Predicted velocity profiles and pen-tip trajectories are shown in figure 7, which illustrate an agreement with experimental data in the case of geometric drawing and Arabic letter.

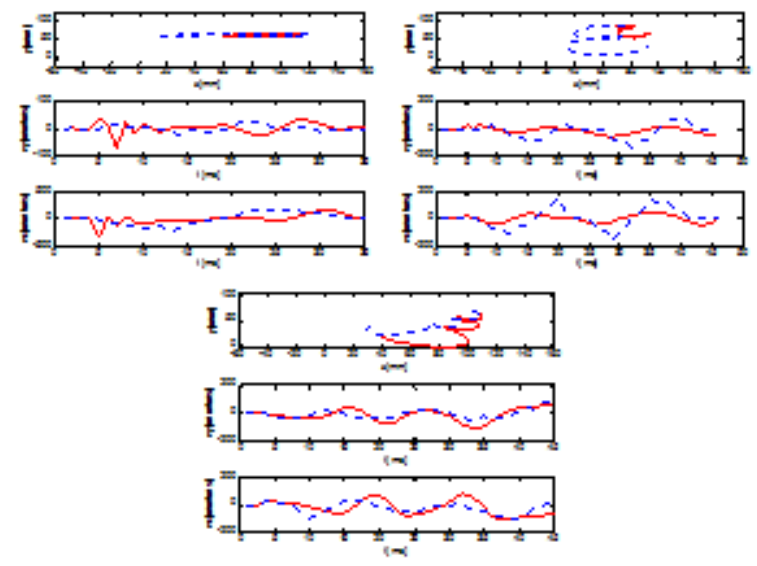

Figure 7. Some validity's results in multiwriter case

Hence, for many kinds of traces (Arabic letter or geometric forms), the proposed model can reconstruct pen-tip traces, from handwriting velocity and $E M G$ forms, with a not negligible error. This depends necessarily on the trace's form, the hand writer's velocity and the writers. Indeed the same individual can have different handwriting velocities, and evidently these velocities differ from one person to another.

\section{THE NOVEL ALGORITHM BASED ON THE BRESENHAM CIRCLE}

In order to explain more this novel approach, we choose the cursive Arabic letter "AYN", Figure 9. We note that this letter is considered as a complex handwriting shape. It's shown that it could be divided into two circles: The first circle is of smaller diameter than the second circle. In this case we were obliged to choose specific coordinates of the main points so that the tracing of two circles could be done.

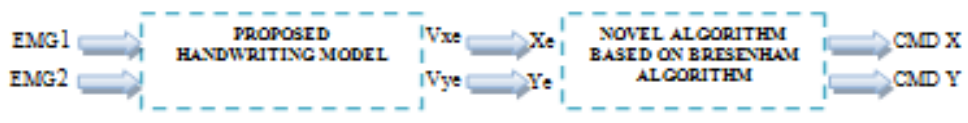

Figure 8. Proposed models

From the coordinates of the letter "AYN" recorded on the data basis of the handwriting shapes, we were able to detect coordinates of the two centers P1(x1, y1) and P2(x2, y2) of each semicircle $\mathrm{C} 1$ and $\mathrm{C} 2$ respectively. We have also detected the coordinates of the beginning and the ending of each arc of circle. To generate the letter "AYN" we proposed two right triangles having as center of gravity both P1 and P2 centers of the circles $\mathrm{C} 1$ and $\mathrm{C} 2$ respectively and that in order to apply the draw circle proposed by Bresenham. Once we have detected the coordinates of the beginning and end of two semicircles, we conducted with the initialization of the vertices of two triangles. The following step was calculating the radius which is the half of the hypotenuse of each triangle so that we would be able to apply the Bresenham circle method us we have mentioned in the previous paragraph, Figure 10. The numerical results are shown in the Figure 11. 


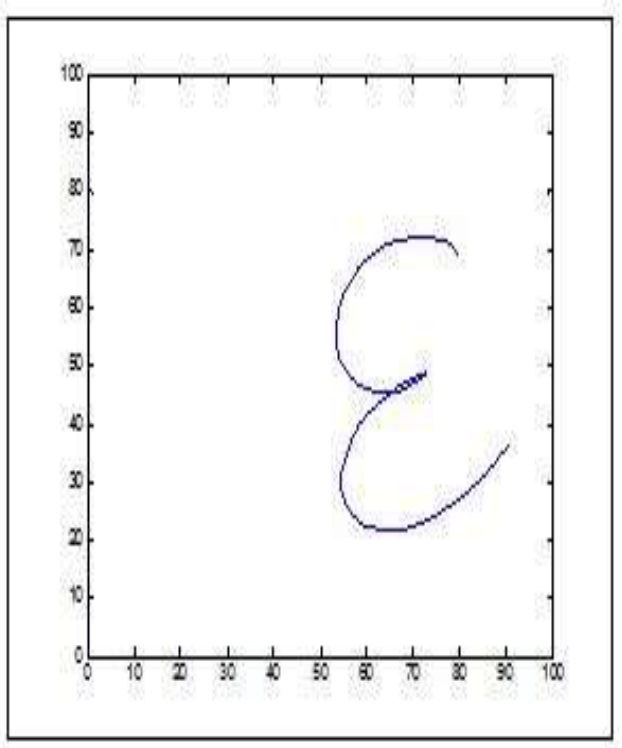

Figure 9. Arabic Letter “AYN"

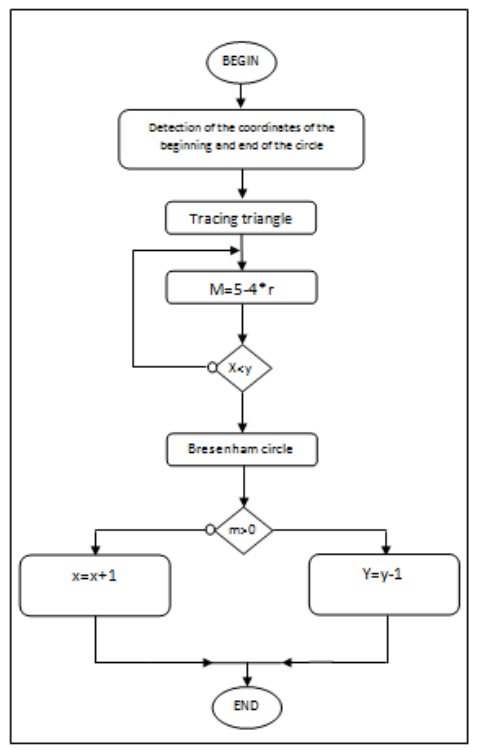

Figure 10. Flow chart for the novel algorithm

As a first example to explain this approach, we chose to create a circle based on the Bresenham algorithm and to compare it to the circle from the database. Figure 4 shows good agreement with the recorded shape and proves that the proposed algorithm can reconstruct the geometric handwriting trace.

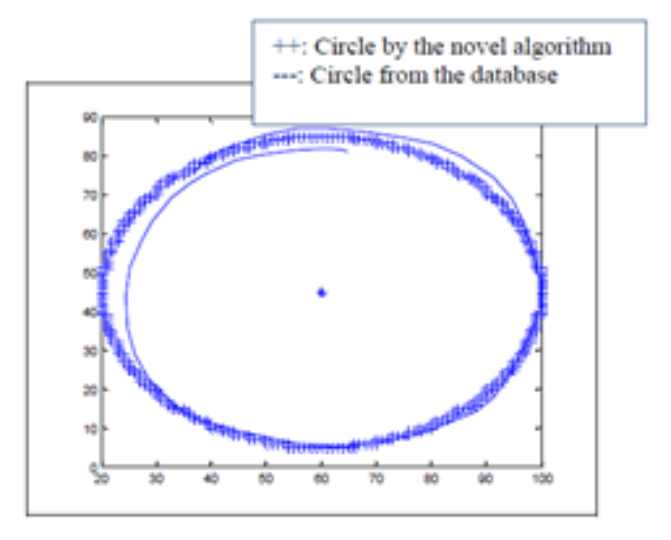

Figure11. Numerical result of circle

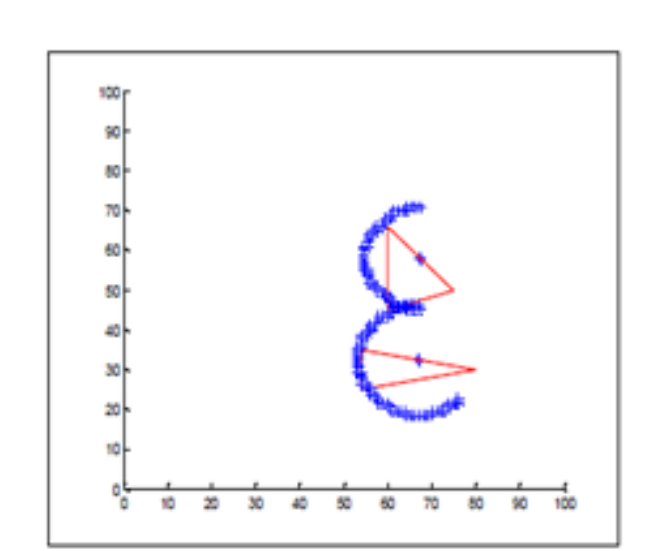

Figure 12. Numerical result of the letter "AYN

\section{DISCUSSION}

We have previously applied the new algorithm to reconstruct three different Arabic letters "AYN" chosen as follow:

- Writer (1)-Example (1): the first letter is generated by writer (1).

- Writer (1)-Example (2): the second is another letter (different than the first) and generated by the same writer (1).

- Writer (2): the third letter is generated by another writer (2).

Figure 13 shows the response of the proposed algorithm in the case of writer (1)-example (1). We remark that the bi-axis algorithm allows to reconstruct the letter but with a considerable error especially in the intermediate area of the letter. 


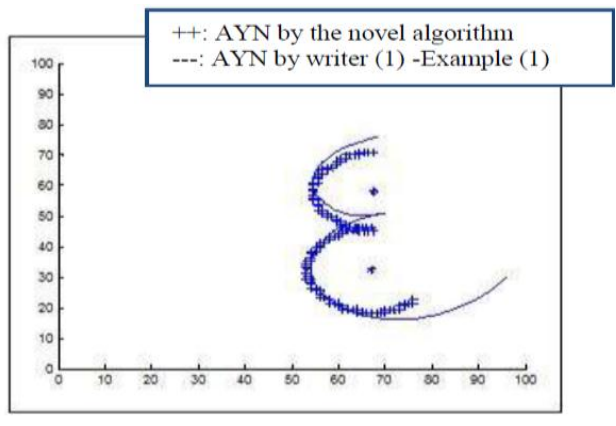

Figure 13. Comparison between the letter "AYN" by the novel algorithm and by writer (1) - example (1)

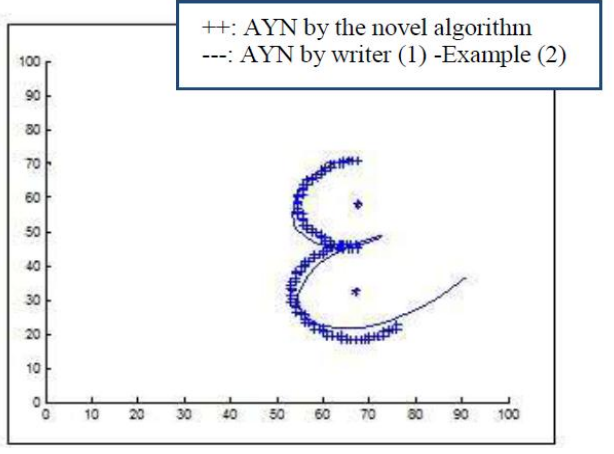

Figure 14. Comparison between the letter "AYN" by the novel algorithm and by writer (1) - example (2)

The second example of the letter "AYN", Figure 14, written by the same writer (1)-example(2), has less curvature in the end of the first circle than the first example (1). Good agreement is shown in this case, but we remark that a little refinement is required.

Figure 15 presents the response of our bi-axis algorithm in the third case (Arabic letter "AYN" written by writer (2)). Despite changing the writer, the proposed bi-axis algorithm can mimic the recorded human handwriting letters.

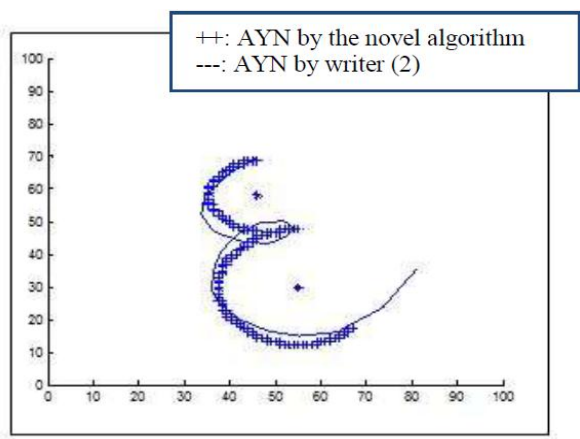

Figure 15. Comparison between the letter "AYN" by the novel algorithm and by writer (2)

To generate another Arabic letter, an amelioration of the proposed algorithm is needed and other parameters are necessary. It was already tested on the "SIN" letter applied on three circles following the same steps of the "AYN"'s algorithm. Figure 16 shows a considerable error between the recorded data and the generated one by the new algorithm. We conclude that amelioration is recommended in the case of very complex handwriting shapes.

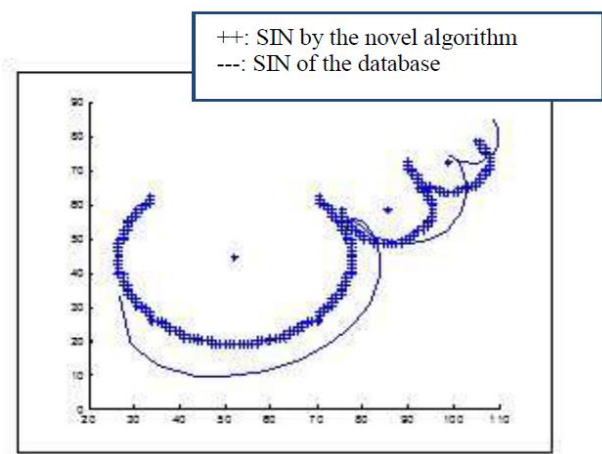

Figure 16. Comparison between the letter "SIN" by the novel algorithm and the database

After the generation of several Arabic letters, the production of Arabic words was the subject of our research. We tried to generate the Arabic surname 'Malek' which presents a series of circle or parts of 
successive circles by applying our new algorithm based on the Bresenham algorithm. Figure 17 shows the generated Arabic word by the new algorithm. We conclude that our novel algorithm can generate Arabic words and mimic human handwritten words.

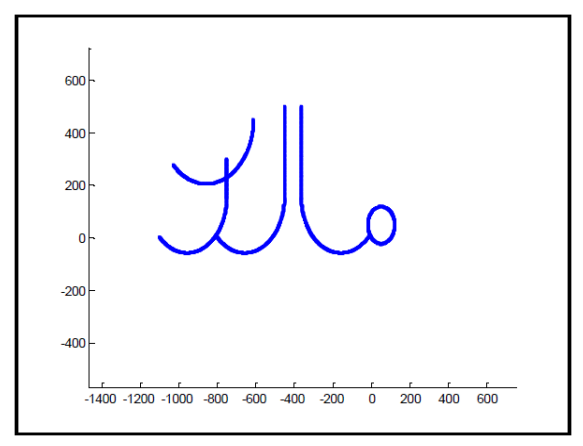

Figure 17. Arabic word generated by the novel algorithm

\section{CONCLUSION}

In this study, the bell shapes shown in the handwriting velocity profile can contain information about the kind of the pen-tip movement. Indeed, a handwriting system is proposed by using of parametric identification technique based on the Recursive Least Square algorithm. The obtained model uses mainly the relationship between the electromyography signals of the forearm muscles and the velocity profile of the pentip moving in $(x, y)$ plane.

According to the results shown in this paper, compatibility is observed in the case of the same writer and the same character. The proposed handwriting velocity model can reconstruct the graphic trace for several Arabic letters and shapes written by the same or different writers, with a not negligible error. As a following step, a new algorithm of generating handwritten geometric forms and Arabic letters, based on the Bresenham bi-axis control algorithm of drawing a circle and using the estimated coordinates previously calculated, is proposed in this article. Given the complexity of the process under study, various steps were taken to generate handwriting shapes. Satisfactory results have been identified and the various responses of the novel bi-axis control algorithm, proposed to mimic the handwriting motion, were conclusive. So it is interesting to continue the improvement of this algorithm to generate other Arabic letters as well as other Arabic words in order to conduct studies for their implementation in the medical field, for the development of a system for the disabled drivers of particular hand. An improvement of the predicted model results must also be made not only in the monowriter case but also in the multiwriter case.

\section{ACKNOWLEDGEMENTS}

So it is interesting to continue the improvement of this algorithm to generate other Arabic letters as well as parts of Arabic words in order to conduct studies for their implementation in the medical field, for the development of a system for the disabled drivers of particular hand.

\section{REFERENCES}

[1] F. Leclerc, Plamondon, R. "Automatic Signature Verification: the State of the Art-1989-1993”, International Journal of Pattern Recognition and Artificial Intelligence, vol. 8, no. 3, pp. 643-660, 1994.

[2] M. Parizeau, Plamondon, R. "A Fuzzy-Syntactic Approach to Allogragh Modeling for Cursive Script Recognition", IEEE Transactions on Pattern Analysis and Machine Intelligence, vol. 17, no. 7, pp. 702-712, 1995.

[3] M. Sugeno, “on stability of fuzzy systems expressed by fuzzy rules with singleton consequents", IEEE Trans. On Fuzzy Systems, vol. 7, no.2, pp. 201-223, 1999.

[4] M. Benrejeb, A. El Abed-Abdelkrim et S. Bel Hadj Ali, "Handwriting process controlled by neural and neurofuzzy IMC approaches", ISCIII Conference, pp. 16-20, Nabeul, 2003.

[5] M. Benrejeb, El Abed-Abdelkrim A., Bel Hadj Ali S., Gasmi M., "A neuro-fuzzy internal model controller for handwriting process", CESA 2003, Lille, 2003. 
[6] A.M. Alimi,; Slimane, F.; Kanoun, S.; Abed, H.E.; Ingold, R.; Hennebert, J. "Arabic Recognition Competition: Multi-font Multi-size Digitally Represented Text”, International Conference on Document Analysis and Recognition (ICDAR), 2011

[7] AM Alimi, M Kherallah, F Bouri, "On-line Arabic handwriting recognition system based on visual encoding and genetic algorithm”, Engineering Applications of Artificial Intelligence, 2009

[8] I. Chihi, A. Abdelkrim and M. Benrejeb, "Analysis of handwriting velocity to identify handwriting processs from electromyographic signals”, American Journal and Applied Sciences, vol. 9, no.10, pp. 1742-1756, 2012

[9] C. J. Kaufman, Rocky Mountain Research Lab., Boulder, CO, private communication, May 1995.

[10] Y. Yorozu, M. Hirano, K. Oka, and Y. Tagawa, "Electron spectroscopy studies on magneto-optical media and plastic substrate interfaces (Translation Journals style)," IEEE Trans 1. J. Magn.Jpn., vol. 2, Aug. 1987, pp. 740741 [Dig. 9th Annu. Conf. Magnetics Japan, 1982, p. 301].

[11] Seima Saki Sl, Sabita Devi, "Design of Intelligent Robotic Arm for Visually Challenged", College Of Engineering And Tech. Coimbatore, India, Proceedings of the 2010 IEEE Students' Technology Symposium, II T Kharagpur, 3-4 April 2010

[12] Biao Zhang, Jianjun Wang, Fuhlbrigge T., "A review of the commercial brain-computer interface technology from perspective of industrial robotics", Automation and Logistics (ICAL), IEEE International Conference, Hong Kong and Macau, 2010.

[13] M. Young, The Techincal Writers Handbook. Mill Valley, CA: University Science, 1989.

[14] J. U. Duncombe, "Infrared navigation-Part I: An assessment of feasibility (Periodical style)," IEEE Trans. Electron Devices, vol. ED-11, pp. 34-39, Jan. 1959.

[15] S. Chen, B. Mulgrew, and P. M. Grant, "A clustering technique for digital communications channel equalization using radial basis function networks," IEEE Trans. Neural Networks, vol. 4, pp. 570-578, July 1993.

[16] R. W. Lucky, “Automatic equalization for digital communication,” Bell Syst. Tech. J., vol. 44, no. 4, pp. 547588, Apr. 1965.

[17] S. P. Bingulac, "On the compatibility of adaptive controllers (Published Conference Proceedings style)," in Proc. 4th Annu. Allerton Conf. Circuits and Systems Theory, New York, 1994, pp. 8-16.

[18] G. R. Faulhaber, "Design of service systems with priority reservation," in Conf. Rec. 1995 IEEE Int. Conf. Communications, pp. 3-8.

[19] W. D. Doyle, "Magnetization reversal in films with biaxial anisotropy," in 1987 Proc. INTERMAG Conf., pp. 2.2-1-2.2-6.

[20] G. W. Juette and L. E. Zeffanella, "Radio noise currents n short sections on bundle conductors (Presented Conference Paper style)," IEEE Summer power Meeting, Dallas, TX, June 22-27, 1990, Paper 90 SM 690-0 PWRS.

[21] J. Williams, "Narrow-band analyzer (Thesis or Dissertation style)," Ph.D. dissertation, Dept. Elect. Eng., Harvard Univ., Cambridge, MA, 1993.

[22] N. Kawasaki, "Parametric study of thermal and chemical nonequilibrium nozzle flow," M.S. thesis, Dept. Electron. Eng., Osaka Univ., Osaka, Japan, 1993.

[23] D.Van Der Gon, Thuring Pr., Strackee J., « A han J. Williams, "Narrow-band analyzer (Thesis or Dissertation style)," Ph.D. dissertation, Dept. Elect. Eng., Harvard Univ., Cambridge, MA, 1993.

[24] N. Kawasaki, "Parametric study of thermal and chemical nonequilibrium nozzle flow," M.S. thesis, Dept. Electron. Eng., Osaka Univ., Osaka, Japan, 1993.

[25] D.Van Der Gon, Thuring Pr., Strackee J., « A handwriting similator», Physiol. Med. Biol., Vol.6, pp.407-414, 1962.

[26] J.S. Mac Donald, "Experimental studies of handwriting signals", Ph. D Dissertation, Mass. Inst. Tech., Cambridge, 1964

[27] M .Yasuhara, "Experimental studies of handwriting process", Rep. Univ. Electro-Comm., 25-2, pp. 233-254, Japon, 1975.

[28] J. P. Wilkinson, "Nonlinear resonant circuit devices (Patent style)," U.S. Patent 3624 12, July 16, 1990.

[29] Iguider Y., Yasuhara M., "Extracting control pulses of handwriting movement", Trans. of the Soc. Inst. and Cont. Eng., Vol.31, №8, pp.1175-1184, Japon, 1995.

[30] Iguider Y., Yasuhara M., "An active recognition of handwritten isolated Arabic charcters", Trans. of the Soc. Inst. and Cont. Eng., Vol.32, Nº, pp.1267-1276, Japon, 1996.

[31] Edelman S., Flash T., "A model of handwriting”, Biological Cybernetics, 57, pp. 25-36, Springer Verlag, 1987.

[32] M. Benrejeb, A. Errachdi, "A new algorithm of neural internal model controller using variable learning rate", International Journal of Neural Networks And Advanced Applications, vol. 1, no. 1, pp. 13-19, 2014

[33] J. E. Bresenham Algorithm for computer control of a digital plotter, IBM Systems Journal, 1965.

[34] N. S. Bernstein, "The co-ordination and regulation of movements", Pergamon Press, Oxford, 1967

[35] Sallagoïty, "Dynamique de coordination spontanée de l'écriture “, Ph. D. Thesis, Université Toulouse III - Paul Sabatier, 2004

[36] L. Castellanos "Statistical Models and Algorithms for Studying Hand and Finger Kinematics and their Neural Mechanisms "Ph. D. Thesis, Carnegie Mellon University Pittsburgh, 2013.

[37] J.M. Inouye1 and F. J. Valer Cuevas "Anthropomorphic tendon-driven robotic hands can exceed human grasping capabilities following optimization" The International Journal of Robotics Research, vol. 0, no. 0, pp. 1-12, 201

[38] G. C. Matrone, C. Cipriani, M. C. Carrozza and G. Magenes, "Real-time myoelectric control of a multi-fingered hand prosthesis using principal components analysis" Journal of NeuroEngineering and Rehabilitation, vol. 9, no. 40, pp. 3-12, 2012. 
[39] G. O. Young, "Synthetic structure of industrial plastics (Book style with paper title and editor)," in Plastics, 2nd ed. vol. 3, J. Peters, Ed. New York: McGraw-Hill, pp. 15-64, 1964.

[40] W.-K. Chen, Linear Networks and Systems (Book style). Belmont, CA: Wadsworth, pp. 123-135 , 1993

[41] I. Chihi, A. Abdlekrim, M. Benrejeb, "Characterization of Electromyography Signals of the Forearm Muscles from Pen-Tip Coordinates, Using RELS Algorithm", International Journal of Emerging Science and Engineering (IJESE) ISSN: 2319-6378, Volume-1, Issue-5, March 2013.

[42] E. H. Miller, "A note on reflector arrays (Periodical style-Accepted for publication)," IEEE Trans. Antennas Propagat., to be published.

[43] M. Sano, Kosaku T., Murata Y. "Modeling of Human Handwriting Motion by Electromyographic Signals on Forearm Muscles”, CCCT'03, Orlando-Florida, 2003..

[44] J. G. Kreifeldt, "An analysis of surface-detected EMG as an amplitude-modulated noise", presented at the 1989 Int. Conf. Medicine and Biological Engineering, Chicago, IL

[45] T. Kosaku, M. Sano, M. Benrejeb, A. El Abed-Abdelkrim "Direct Linear Models from Forearm Electromyographic Signals to Pen-Tip Movement in Handwriting Process".

[46] J. Wang, "Fundamentals of erbium-doped fiber amplifiers arrays (Periodical style—Submitted for publication)," IEEE J. Quantum Electron., submitted for publication, 2014.

[47] R. G. J. Meunlenbroek et A. J. W. M. Thomassen "Stroke-direction preferences in drawing and handwriting, Human Movement Science", Vol. 10, pp. 247-270, 1991.

[48] W. Ning, C. Peng, “A Novel Method for Projection Based on Bresenham Like Algorithm”, International Conference on Intelligent Systems Design and Engineering Application, 2012.

[49] J.S. Mac Donald, "Experimental studies of handwriting signals", Ph. D Dissertation, Mass. Inst. Tech., Cambridge, 1964

[50] M .Yasuhara, "Experimental studies of handwriting process", Rep. Univ. Electro-Comm., 25-2, pp. 233-254, Japon, 1975.

[51] J. P. Wilkinson, "Nonlinear resonant circuit devices (Patent style)," U.S. Patent 3624 12, July 16, 1990.

[52] Iguider Y., Yasuhara M., "Extracting control pulses of handwriting movement", Trans. of the Soc. Inst. and Cont.Eng., Vol.31, Nº, pp.1175-1184, Japon, 1995.

[53] Iguider Y., Yasuhara M., "An active recognition of handwritten isolated Arabic charcters", Trans. of the Soc. Inst.and Cont. Eng., Vol.32, Nº, pp.1267-1276, Japon, 1996.

[54] Edelman S., Flash T., "A model of handwriting”, Biological Cybernetics, 57, pp. 25-36, Springer Verlag, 1987.

[55] M. Benrejeb, A. Errachdi, "A new algorithm of neural internal model controller using variable learning rate", International Journal of Neural Networks And Advanced Applications, vol. 1, no. 1, pp. 13-19, 2014

[56] J. E. Bresenham Algorithm for computer control of a digital plotter, IBM Systems Journal, 1965.

[57] N. S. Bernstein, "The co-ordination and regulation of movements", Pergamon Press, Oxford, 1967

[58] Sallagoïty, "Dynamique de coordination spontanée de l'écriture", Ph. D. Thesis, Université Toulouse III - Paul Sabatier, 2004.

[59] L. Castellanos "Statistical Models and Algorithms for Studying Hand and Finger Kinematics and their Neural Mechanisms "Ph. D. Thesis, Carnegie Mellon University Pittsburgh, 2013.

[60] J.M. Inouye1 and F. J. Valer Cuevas "Anthropomorphic tendon-driven robotic hands can exceed human grasping capabilities following optimization" The International Journal of Robotics Research, vol. 0, no. 0, pp. 1-12, 201

[61] G. C. Matrone, C. Cipriani, M. C. Carrozza and G. Magenes, "Real-time myoelectric control of a multi-fingered hand prosthesis using principal components analysis" Journal of NeuroEngineering and Rehabilitation, vol. 9, no. 40, pp. 3-12, 2012.

[62] G. O. Young, "Synthetic structure of industrial plastics (Book style with paper title and editor)," in Plastics, 2nd ed. vol. 3, J. Peters, Ed. New York: McGraw-Hill, pp. 15-64, 1964.

[63] W.-K. Chen, Linear Networks and Systems (Book style). Belmont,CA: Wadsworth, pp. 123-135 , 1993

[64] I. Chihi, A. Abdlekrim, M. Benrejeb, "Characterization of Electromyography Signals of the Forearm Muscles from Pen-Tip Coordinates, Using RELS Algorithm”, International Journal of Emerging Science and Engineering (IJESE) ISSN: 2319-6378, Volume-1, Issue-5, March 2013.

[65] E. H. Miller, "A note on reflector arrays (Periodical style-Accepted for publication)," IEEE Trans. Antennas Propagat., to be published.

[66] M. Sano, Kosaku T., Murata Y. "Modeling of Human Handwriting Motion by Electromyographic Signals on Forearm Muscles”, CCCT’03, Orlando-Florida, 2003.

[67] J. G. Kreifeldt, “An analysis of surface-detected EMG as an amplitude-modulated noise," presented at the 1989 International Conference Medicine and Biological Engineering, Chicago, IL

[68] T. Kosaku, M. Sano, M. Benrejeb, A. El Abed-Abdelkrim "Direct Linear Models from Forearm Electromyographic Signals to Pen-Tip Movement in Handwriting Process".

[69] J. Wang, "Fundamentals of erbium-doped fiber amplifiers arrays (Periodical style—Submitted for publication)," IEEE J. Quantum Electron., submitted for publication, 2014.

[70] R. G. J. Meunlenbroek et A. J. W. M. Thomassen "Stroke-direction preferences in drawing and handwriting, Human Movement Science", Vol. 10, pp. 247-270, 1991.

[71] W. Ning, C. Peng, "A Novel Method for Projection Based on Bresenham Like Algorithm", International Conference on Intelligent Systems Design and Engineering Application, 2012. 